Report 99-12 (Revised)

November, 1999

\title{
HIGHER-DERIVATIVE QUANTUM COSMOLOGY
}

\author{
Simon Davis \\ School of Mathematics and Statistics \\ University of Sydney \\ NSW 2006
}

\begin{abstract}
The quantum cosmology of a higher-derivative gravity theory arising from the heterotic string effective action is reviewed. A new type of Wheeler-DeWitt equation is obtained when the dilaton is coupled to the quadratic curvature terms. Techniques for solving the Wheeler-DeWitt equation with appropriate boundary conditions shall be described, and implications for semiclassical theories of inflationary cosmology will be outlined.
\end{abstract}

Current version of the text of a talk given at ACGRG2, 9 July 1998

AMS Classification: 83-06, 83C45, 83E30, 83F05 


\section{Higher-Derivative Gravity Theories}

Since the renormalization of the Einstein-Hilbert action for general relativity introduces higher-order curvature terms, it has long been expected that the development of quantum cosmology would include higher-derivative gravity theories. Further support for this approach arises from the presence of quadratic and higher-order curvature terms in string effective actions, where modifications to the Ricci scalar are required for maintaining conformal invariance of the sigma model, describing string propogation in a given background, even after inclusion of quantum corrections to the $\beta$-function.

Typically, one may consider actions of the form

$$
I=\int d^{4} x \sqrt{-g}\left(R+c_{1}\left(g_{\mu \nu}, \phi, \chi_{\gamma}, \ldots\right)\left(R_{\mu \nu \rho \sigma} R^{\mu \nu \rho \sigma}+\alpha R_{\mu \nu} R^{\mu \nu}+\beta R^{2}\right)+O\left(R^{3}\right)\right)
$$

and field-dependence of the coefficients $c_{1}, \ldots$ provides couplings between matter fields and the curvature.

There are several types of higher-derivative gravity theories selected by physical considerations:

(i) Lovelock gravity required for unitarity and elimination of ghosts

Amongst the curvature invariants that arise in superstring effective actions are the dimensionally continued Lovelock invariants

$$
\left.L_{(n)}=\frac{(2 n) !}{2^{n}} R_{\left[i_{1} i_{2}\right.}^{i_{1} i_{2}} R_{i_{3} i_{4}}{ }^{i_{3} i_{4}} \ldots R_{i_{2 n-1} i_{2 n}}\right]^{i_{2 n-1} i_{2 n}}
$$

which vanish in ten dimensions when $n \geq 6$. As generalizations of the Gauss-Bonnet invariant, they are the only combinations of Riemann tensors that give rise to secondorder field equations for the metric. In a sigma-model with expansion parameter $\alpha^{\prime}$, these curvature terms would occur at order $O\left(\alpha^{n-1}\right)$ in the effective action. The elimination of ghost fields in higher-order gravitational actions is achieved by eliminating terms of the type $h \nabla^{2 n} h, n \geq 2$, where $h_{\mu \nu}$ is the perturbation about the flat-space metric, through metric redefinitions. The linearized equation for the graviton field is then second-order, possessing physically consistent stable classical solutions, in contrast to equations with third and higher-order derivatives of the metric, and at the quantum level, the ghost poles will cancel in the graviton propagator. The only non-zero Lovelock terms that could appear in ten-dimensional superstring effective actions are $L_{(0)}, L_{(1)}, L_{(2)}, L_{(3)}, L_{(4)}, L_{(5)}$ and functions of these tensors. While $L_{(5)}$ is a topological invariant in ten dimensions, the other three curvature combination $L_{(2)}, L_{(3)}, L_{(4)}$ contain dynamical higher-derivative terms. 
(ii) Superstring effective actions with higher-order curvature terms

There is a particular combination of Lovelock invariants which is equivalent to a ChernSimons theory with $\mathrm{SO}(1,9)$ gauge group [1], and this suggests the existence of a tendimensional superstring theory reducing to an effective field theory containing $\mathrm{D}=10$ superYang-Mills theory with gauge group $\mathrm{SO}(1,9)$, combined with topological terms, as an appropriate modification of general relativity with improved renormalizability properties.

In this superstring effective action, the curvature terms are generated by identifying the gauge potential with the metric connection. The various different quartic curvature combinations that can be included in low-energy superstring and heterotic effective string actions are restricted by their consistency with supersymmetry [2][3].

(iii) Born-Infeld actions

Born-Infeld effective actions, which typically involve determinants of two-index fields such as the field strength $F_{\mu \nu}$ or the Riemann tensor $R_{\mu \nu}$ may represent a viable alternative to the expansion of the effective action in powers of $\alpha^{\prime}$, relevant for specific physical configurations. Difficulties associated with the infinite expansion in powers of $\alpha^{\prime}$ at the Planck scale might then be circumvented.

\section{Classical Cosmological Solutions of Equations of Motion of Higher-Derivative Theories}

Since solutions to the general relativistic field equations contain initial curvature singularities whenever the dominant energy condition is satisfied, one of the motivations for developing quantum cosmology has been the theoretical justification of the absence of the singularity. Non-singular solutions may arise in theories which are modifications of general relativity, and classical cosmological solutions to the equations of motion for several different types of theories containing higher-order curvature terms have been analyzed with regard to the absence of singularities and the existence of de Sitter phases.

(i) Singularity-free cosmological solutions of heterotic string effective actions with quadratic curvature terms

An action, which combines higher-derivative gravity with a scalar field, has been shown to have singularity-free cosmological solutions [4][5]. At string tree-level and first-order in the $\alpha^{\prime}$-expansion of the compactified heterotic string effective action in four dimensions, the dynamics of the graviton, dilaton field $\mathrm{S}$ and modulus field $\mathrm{T}$ can be described by

$$
L_{e f f .}=\frac{1}{2 \kappa^{2}} R+\frac{D S D \bar{S}}{(S+\bar{S})^{2}}+3 \frac{D T D \bar{T}}{(T+\bar{T})^{2}}+\frac{1}{8}(\operatorname{Re} S) R_{G B}^{2}+\frac{1}{8}(\operatorname{Im} S) R \tilde{R}
$$


The couplings for the quadratic curvature terms are dimensionless, and this is consistent with the $\alpha^{\prime}$ expansion because $\frac{\alpha^{\prime}}{\kappa^{2}}$ has no units in four dimensions. If $\operatorname{Re} T$, representing the square of the compactification radius, is set equal to a constant, and $\operatorname{Im} T=0$, the kinetic term for the modulus field vanishes. In addition, defining the real part of the dilaton field to be $\operatorname{Re} S=\frac{1}{g_{4}^{2}} e^{\Phi}$, setting $\operatorname{Im} S$ equal to zero and choosing units such that $\kappa=1$ and the effective action can be set equal to

$$
I=\int d^{4} x \sqrt{-g}\left[R+\frac{1}{2}(D \Phi)^{2}+\frac{e^{\Phi}}{4 g_{4}^{2}}\left(R_{\mu \nu \kappa \lambda} R^{\mu \nu \kappa \lambda}-4 R_{\mu \nu} R^{\mu \nu}+R^{2}\right)\right]
$$

This action is also obtained when $\operatorname{Im} S=$ constant and the background geometry is restricted to have a Friedmann-Robertson-Walker metric. The Gauss-Bonnet invariant arises in this action, but it is multiplied now by the factor $\frac{e^{\Phi}}{4 g_{4}^{2}}$, where $g_{4}$ is the four dimensional string coupling constant and $\Phi$ is a scalar field, so that the integral is not a topological invariant.

In the phase space of classical solutions to the field equations of the four-dimensional action (4), there is a class of space-times which have no singularity for a large range of values of the dilaton and modulus fields.

(ii) Dimensionally-continued Euler actions and Lovelock gravity theories

The study of cosmological solutions of Kaluza-Klein theories [6] and string effective actions has led to investigations of dimensionally-continued Euler actions and Lovelock theories [7][8]. The direct product of a four-dimensional Friedmann-Robertson-Walker metric and a metric for a compact six-dimensional space with a second scale factor is postulated. The gravitational field equations may be solved to obtain the time-dependence of the scale factors. The geodesic completeness of cosmological solutions to Kaluza-Klein theories with quadratic curvature terms has also been investigated [9].

(iii) $R^{2}$ inflation and higher-derivative gravity without scalar fields

An early analysis in the previous decade of $R^{2}$ theories [10] and $C^{2}$ theories [11] showed that an $R^{2}$ term leads to particle production and inflation with minimal dependence on the initial conditions. The $C^{2}$ term gives rise to large anisotropy [12] and the destabilization of positive $\Lambda$ metrics. Inflation has also been derived from higher-derivative terms directly obtained as renormalization counterterms [13][14] without the inclusion of scalar or inflaton fields. 
(iv) Higher-derivative gravity with the dilaton field

Dilaton fields have been considered useful for the cosmology to exhibit inflation. It is known that most classical string equations of motion do not lead to inflation [15]. A set of higher-derivative gravity theories with a dilaton field has been investigated and shown to produce the required inflationary growth of the Friedmann-Robertson-Walker scale factor $[16]$.

(v) Cosmology of M-type theories

More recently, the low-energy effective actions derived with the higher-dimensional Mtheories have been studied [17][18]. The actions generally involve the coupling of standard gravity to scalar fields with a potential determined by the theory, although the analysis could be extended to include higher-orders curvature terms. Classical cosmological solutions have been obtained, by compactifying on flat or maximally symmetric subspaces [17], and their singularity structure has been determined.

\section{Quantum Cosmology for Gravity Plus Higher-Order Terms}

Much of the initial work on higher-derivative quantum cosmology has been developed with only curvature terms and no scalar field in the action. The quantum cosmology of standard gravity coupled to a scalar field has been investigated by many authors [19]-[22]. These techniques have been adapted to string cosmology, based on an effective action consisting of the Ricci scalar, dilaton field and antisymmetric 3-index field [23]-[27]. The non-zero vacuum expectation value of the scalar field in the potentials arising in grand unified theories drives inflation in semi-classical cosmology and again it is found to be useful in obtaining wave functions representing inflationary solutions in quantum cosmology.

(i) Without scalar fields

The quantum cosmology of superstring and heterotic string effective actions in ten dimensions with higher-derivative curvature terms up to fourth order also has been investigated [28][29]. The Wheeler-DeWitt equations for both theories, in the mini-superspace of metrics with different scale factors for the physical and internal spaces, differ significantly from the equation obtained for the higher-derivative theory considered in this paper because of the coupling of the curvature and scalar field. For the superstring, the differential equation is not generally solvable by analytic methods, and it is only reducible to the form of a diffusion equation when the curvatures of the physical and internal spaces are set equal to zero, and the scalar field is set equal to a constant. 
(ii) With scalar fields

The coupling of scalar fields to higher-derivative curvature terms in string effective actions results in Hamiltonians which are not quadratic function of the momenta. Even when there are only quadratic curvature terms in the heterotic string effective action, the Hamiltonian cannot be expressed as a simple function of the canonical momenta, preventing a derivation of the Wheeler-DeWitt equation [29]. The derivatives of the coordinate fields for the model studied later in this paper are also found to be given by expressions containing fractional roots and inverses of canonical momenta. However, it is shown that resulting equation can be converted to a partial differential equation, resolving the problem of deriving a Wheeler-DeWitt equation.

Higher-order terms in the effective action will give rise to corrections in the theoretical predictions for the inflationary epoch. Given a fundamental theory at Planck scale with higher-order terms, it is appropriate to consider a boundary located between the Planck era and the inflationary epoch where the predictions of quantum cosmology of the higher-derivative theory could be matched, in principle, to the predictions of the quantum theory of standard gravity coupled to matter fields. The inclusion of this boundary will have an effect on both the quantum cosmology of the more fundamental theory and the computations of the standard model.

(iii) $f(R)$ theories

The quantum cosmology of $f(R)$ theories can be contrasted with that of quadratic gravity theories [30]. The essential simplification in the study of these theories is a conformal transformation which maps the $f(R)$ theory to an Einstein-Hilbert action coupled to a scalar field. A cubic curvature term, for example, has been found to lead to the existence of a region in parameter space for which neither the no-boundary or tunneling boundary conditions produce an inflationary growth that simultaneously resolves the horizon and flatness problems.

\section{Supersymmetric Quantum Cosmology with Higher-Derivative Terms}

The model (4) containing quadratic curvature terms and the dilaton field can be quantized and the Hamiltonian constraint would be given by the Wheeler-DeWitt equation. Since the solution to this equation generally requires a reduction in the number of degrees of freedom in the metric field, it is convenient to consider only a minisuperspace of Friedmann-Robertson-Walker metrics with $K=1$ (closed model), $K=0$ (spatially flat 
model) or $K=-1$ (open model). The minisuperspace action is then

$$
\begin{aligned}
I=\int & d^{4} x a^{3}(t) \frac{r^{2} \sin \theta}{\left(1-K r^{2}\right)^{\frac{1}{2}}}\left[6 a^{-2}\left(a \ddot{a}+\dot{a}^{2}+K\right)+\frac{1}{2}(D \Phi)^{2}\right. \\
& \left.+\frac{e^{\Phi}}{4 g_{4}^{2}}\left(-18 \frac{\ddot{a}^{2}}{a}-\frac{6}{a^{4}}\left(a \ddot{a}+2 \dot{a}^{2}+2 K\right)^{2}+24 a^{-2}\left(\ddot{a}+\frac{\dot{a}^{2}}{a}+\frac{K}{a}\right)^{2}\right)\right]
\end{aligned}
$$

When a boundary is placed for $K=1$, and the action reduces to a one-dimensional integral

$$
I=\bar{V} \int d t\left[\left(6 a^{2} \ddot{a}+6 a \dot{a}^{2}+6 a K\right)+\frac{1}{2} a^{3}(D \Phi)^{2}+6 \frac{e^{\Phi}}{4 g_{4}^{2}} \ddot{a}\left(\dot{a}^{2}+K\right)\right]
$$

where $\bar{V}$ is a time-independent volume factor, which is given by $\frac{V\left(t_{f}\right)}{a^{3}\left(t_{f}\right)}$, where $V\left(t_{f}\right)$ is the volume of the three-dimensional spatial hypersurface at a fixed final time $t_{f}$.

The one-dimensional integral (6) is an example of an action of the following type

$$
I=\int d t L\left(t, y, \dot{y}, \ldots, y^{(m)}, z, \dot{z}, \ldots, z^{(n)}\right)
$$

The conjugate momenta are defined to be

$$
\begin{aligned}
p_{1} & =\frac{\partial L}{\partial \dot{y}}-\frac{d}{d t}\left(\frac{\partial L}{\partial \ddot{y}}\right)+\ldots+(-1)^{m-1} \frac{d^{m-1}}{d t^{m-1}}\left(\frac{\partial L}{\partial y^{(m)}}\right) \\
p_{2} & =\frac{\partial L}{\partial \ddot{y}}-\frac{d}{d t}\left(\frac{\partial L}{\partial y^{(3)}}\right)+\ldots+(-1)^{m-2} \frac{d^{m-2}}{d t^{m-2}}\left(\frac{\partial L}{\partial y^{(m)}}\right) \\
& \vdots \\
p_{m} & = \\
p_{m+1} & =\frac{\partial L}{\partial \dot{z}}-\frac{d}{d t}\left(\frac{\partial L}{\partial \ddot{z}}\right)+\ldots+(-1)^{n-1} \frac{d^{n-1}}{d t^{n-1}}\left(\frac{\partial L}{\partial y^{(n)}}\right) \\
& \vdots \\
p_{m+n} & =
\end{aligned}
$$

In particular, for the Lagrangian $L(t, a, \dot{a}, \ddot{a}, \Phi)$ we have

$$
\begin{aligned}
& p_{1}=\frac{\partial L}{\partial \dot{a}}-\frac{d}{d t}\left(\frac{\partial L}{\partial \ddot{a}}\right)=-\frac{6}{g_{4}^{2}} e^{\Phi} \dot{\Phi}\left(\dot{a}^{2}+K\right) \equiv P_{a} \\
& p_{2}=\frac{\partial L}{\partial \ddot{a}} \\
& p_{3}=\frac{\partial L}{\partial \dot{\Phi}}=a^{3} \dot{\Phi} \equiv P_{\Phi}
\end{aligned}
$$


Given the conjugate momenta to $q_{1}=a, q_{2}=\dot{a}$ and $q_{3}=\Phi$ using the Ostrogadski method for higher-derivative actions [31][32][33], we find that the Hamiltonian is

$$
\begin{aligned}
H & =p_{1} \dot{q}_{1}+p_{2} \dot{q}_{2}+p_{3} \dot{q}_{3}-L \\
& =-6 a\left(\dot{a}^{2}+K\right)-6 \frac{e^{\Phi}}{g_{4}^{2}} \dot{\Phi}\left(\dot{a}^{2}+K\right) \dot{a}+a^{3} \dot{\Phi}^{2}-\frac{1}{2} a^{3}(D \Phi)^{2} \\
& =-g_{4}^{2} P_{\Phi}^{-1} e^{-\Phi} a^{4} P_{a}+\frac{1}{2 a^{3}} P_{\Phi}^{2}-\left[\frac{g_{4}^{2}}{6} P_{\Phi}^{-1} e^{-\Phi} P_{a}^{2} a^{3} P_{a}-K P_{a}^{2}\right]^{\frac{1}{2}}
\end{aligned}
$$

given the homogeneity of the scalar field. In a Lorentzian space-time, a differential operator is obtained by making the substitutions

$P_{a} \rightarrow-i \frac{\partial}{\partial a}$ and $P_{\Phi} \rightarrow-i \frac{\partial}{\partial \Phi}$.

The Wheeler-DeWitt equation $H \Psi=0$ is an pseudo-differential equation, which can be transformed into a sixth-order partial differential equation.

$$
\begin{aligned}
-\frac{g_{4}^{2}}{6} e^{\Phi} & \left(a^{3} \frac{\partial^{4} \Psi}{\partial a^{3} \partial \Phi}+6 a^{2} \frac{\partial^{3} \Psi}{\partial a^{2} \partial \Phi}\right)+K e^{2 \Phi}\left(\frac{\partial^{3} \Psi}{\partial a^{2} \partial \Phi}+\frac{\partial^{4} \Psi}{\partial a^{2} \partial \Phi^{2}}\right) \\
& =a^{4} g_{4}^{4}\left[4 a^{3} \frac{\partial \Psi}{\partial a}+a^{4} \frac{\partial^{2} \Psi}{\partial a^{2}}\right]+a g_{4}^{2} e^{\Phi}\left(\frac{\partial^{4} \Psi}{\partial a \partial \Phi^{3}}-\frac{\partial^{3} \Psi}{\partial a \partial \Phi^{2}}\right) \\
& +\frac{3}{2} g_{4}^{2} e^{\Phi}\left(a \frac{\partial^{2} \Psi}{\partial a \partial \Phi}-\frac{\partial^{3} \Psi}{\partial \Phi^{3}}\right)+\frac{1}{4 a^{6}} e^{\Phi} \frac{\partial}{\partial \Phi}\left(e^{\Phi} \frac{\partial^{5} \Psi}{\partial \Phi^{5}}\right)
\end{aligned}
$$

Point symmetries of this equation may be checked with the Kersten programme [34], which can be used when the coefficients multiplying the derivatives are polynomial functions of the independent variables, and equation (10) can be cast in the following form through the change of variables $w=e^{\Phi}$ :

$$
\begin{aligned}
-\frac{g_{4}^{2}}{6} w^{2} & \left(a^{3} \frac{\partial^{4} \Psi}{a^{2} \partial a^{3} \partial w}+6 a^{2} \frac{\partial^{3} \Psi}{\partial a^{3}}\right)+K w^{3}\left(2 \frac{\partial^{2} \Psi}{\partial a^{2}}+3 \frac{\partial^{3} \Psi}{\partial a^{2} \partial \Phi}+\frac{\partial^{4} \Psi}{\partial a^{2} \partial \Phi^{2}}\right) \\
= & a^{4} g_{4}^{4}\left[4 a^{3} \frac{\partial \Psi}{\partial a}+a^{4} \frac{\partial \Psi}{\partial a^{2}}\right]+a g_{4}^{2} w^{3}\left(2 \frac{\partial^{3} \Psi}{\partial a \partial w^{2}}+w \frac{\partial^{4} \Psi}{\partial a \partial w^{3}}\right) \\
+ & \frac{3}{2} a g_{4}^{2} w^{2} \frac{\partial^{2} \Psi}{\partial a \partial w}-\frac{3}{2} g_{4}^{2} w^{2}\left(\frac{\partial \Psi}{\partial w}+3 w \frac{\partial^{2} \Psi}{\partial w^{2}}+w^{2} \frac{\partial^{3} \Psi}{\partial w^{3}}\right) \\
+ & \frac{1}{4 a^{6}}\left(w^{8} \frac{\partial^{6} \Psi}{\partial w^{6}}+15 w^{7} \frac{\partial^{5} \Psi}{\partial w^{5}}+65 w^{6} \frac{\partial^{4} \Psi}{\partial w^{4}}+91 w^{5} \frac{\partial^{3} \Psi}{\partial w^{3}}+34 w^{4} \frac{\partial^{2} \Psi}{\partial w^{2}}\right. \\
& \left.+2 w^{3} \frac{\partial \Psi}{\partial w}\right)
\end{aligned}
$$


The Laplace transform with respect to the variable $\Phi$ gives a mixed differencedifferential equation in $a$ and the transform parameter $s$. Denoting the Laplace transform of $\Psi(\Phi, a)$ by $\psi(s, a)$, it follows that a second-order recurrence operator in $s$ and a thirdorder differential operator in $a$ act on the transform of the wave function.

$$
\begin{aligned}
-\frac{g_{4}^{2}}{6} a^{3} & {\left[(s-1) \frac{d^{3} \psi(s-1, a)}{d a^{3}}-\frac{d^{3} \Psi(0, a)}{d a^{3}}\right]-g_{4}^{2} a^{2}\left[(s-1) \frac{d^{2} \psi(s-1, a)}{d a^{2}}-\frac{d^{2} \Psi(0, a)}{d a^{2}}\right] } \\
& +K\left[(s-1)(s-2) \frac{d^{2} \psi(s-2, a)}{d a^{2}}-(s-1) \frac{d^{2} \Psi(0, a)}{d a^{2}}-\frac{d^{2} \Psi^{\prime}(0, a)}{d a^{2}}\right] \\
= & a^{4} g_{4}^{4}\left[4 a^{3} \frac{d \psi(s, a)}{d a}+a^{4} \frac{d^{2} \psi(s, a)}{d a^{2}}\right] \\
& +a g_{4}^{2}\left[(s-1)^{2}(s-2) \frac{d \psi(s-1, a)}{d a}-(s-1)(s-2) \frac{d \Psi(0, a)}{d a}\right. \\
& +\frac{3}{2} g_{4}^{2} a\left[(s-1) \frac{d \psi(s-1, a)}{d a}-\frac{d \Psi(0, a)}{d a}\right] \\
& -\frac{3}{2} g_{4}^{2}\left[(s-1)^{3} \psi(s-1, a)-(s-1)^{2} \Psi(0, a)-(s-1) \Psi^{\prime}(0, a)-\Psi^{\prime \prime}(0, a)\right] \\
+ & \frac{1}{4 a^{6}}\left[(s-2)^{5}(s-1) \psi(s-2, a)-(s-2)^{4}(s-1) \Psi(0, a)\right. \\
& -(s-2)^{3}(s-1) \Psi^{\prime}(0, a)-(s-2)^{2}(s-1) \Psi^{\prime \prime}(0, a)-(s-2)(s-1) \Psi^{\prime \prime \prime}(0, a) \\
& \\
&
\end{aligned}
$$

The mixed difference-differential equation can be solved by considering the differential and recursion operators separately. Solving first the recursion relation gives rise to a higher-order ordinary differential equation in $a$. Arbitrary parameters are determined by specifying values of $\Psi(0, a)$ and $\Psi\left(\Phi_{0}, a\right), \Phi_{0} \gg 1$ and derivatives up to fifth order in $\Phi$. The value $\Phi=0$ leads to a vanishing kinetic term for the dilaton field and the quadratic curvature term being a topological invariant, so that $\Psi(0, a)$ should equal the wave function in the minisuperspace of Friedmann-Robertson-Walker metrics in a theory of pure gravity.

The path integral which defines the wave function does not converge when the action in the weighting factor represents Einstein gravity coupled to a scalar field. One may anticipate that a higher-derivative action can be embedded in a renormalizable theory, and that the evaluation of the path integral with different initial data, determined by the Hartle-Hawking or tunneling boundary condition, produces a wave function which then could be compared with inflationary cosmology. This comparison can be used to select the most appropriate boundary condition. 
Standard inflationary cosmology might receive corrections from two sources. First, the inclusion of graviton loops will alter the perturbative calculations. Secondly, it is appropriate to specify a boundary between the Planck era and the inflationary epoch and to study quantum effects on a manifold with a boundary. The boundary will affect the range of the transform variable in the momentum space representation, and extrinsic curvature terms will be relevant for the quantum theory.

The same techniques can be applied to other superstring effective actions with higherorder curvature terms or theories derived from the recently-developed M-theories. The conjugate momenta, the Hamiltonian and the Wheeler-DeWitt equation may be derived, and the solutions to the partial differential equation for $\Psi$ can be obtained. Coupling of the scalar field to the curvature tensor should generally lead to a form of the Hamiltonian containing inverse or fractional powers of the momenta. When the coefficients of the derivatives in the Wheeler-DeWitt equation are exponential functions, the Laplace transform can be used to reduce the differential equation to a mixed difference-differential equation. Thus, the form of superstring effective actions containing higher-order terms with couplings involving exponential functions is conducive to the reduction of the WheelerDeWitt equation to an equation with derivatives of fewer variables. If solutions to the Wheeler-DeWitt equation for a consistent unified theory of gravity and the elementary interactions can be obtained, they may represent wave functions adequately describing cosmology both at Planck scales and during the inflationary epoch.

\section{Acknowledgements}

I would like to thank P. Zeitsch for running the Kersten program on a sixth-order differential equation similar to the Wheeler-DeWitt equation and Dr H. C. Luckock for useful discussions regarding quantization of higher-derivative theories. Equations (10) - (13) have

been altered from the form given in the talk because the inclusion of a factor of $a^{3}$ in the momentum $P_{\Phi}$ gives rise to a different expression for the Hamiltonian and the resulting Wheeler-DeWitt equation. Research on this project has been supported by an ARC Small Grant.

\section{References}

[1] F. Müller-Hoissen, Ann. der Physik 7 (1991) 543 - 557

[2] M. de Roo, H. Suelmann and A. Weidmann, Phys. Lett. $\underline{280 B}$ (1992) 39 - 46

[3] H. Suelmann, Int. J. Mod. Physics D, Vol. 3, No. 1 (1994) 285 - 288

[4] I. Antoniadis, J. Rizos and K. Tamvakis, Nucl. Phys. $\underline{415 B}$ (1994) 497 - 514

[5] P. Kanti, J. Rizos and K. Tamvakis, Phys. Rev. D59 (1999) 083512

[6] F. Müller-Hoissen, Classical Quant. Grav. $\underline{3}$ (1986) 665 - 682 
[7] J. Demaret, Y. De Rop, P. Tombal and A. Moussiaux, Gen. Rel. Grav. 24(1992) 1169 - 1183.

[8] G. A. Mena Marugin, Phys. Rev. D42 (1990) 2607 - 2620

[9] K. Kleidis and D. B. Papadopoulos, J. Math. Phys. $\underline{38(6)}$ (1997) 3189 - 3208

[10] M. B. Mijic, M. S. Morris, W.-M. Suen, Phys. Rev. D34 (1986) 2934 - 2946

M. B. Mijic, M. S. Morris, W.-M. Suen, Phys. Rev. D39 (1989) 1496 - 1510

M. S. Morris, Phys. Rev. D39 (1989) 1511 - 1516

[11] A. Berkin, Phys. Rev. D $\underline{D 44}$ (1991) 1020 - 1027

[12] S. W. Hawking and J. C. Luttrell, Nucl. Phys. B247 (1984) 250 - 265

S. W. Hawking and J. C. Luttrell, Phys. Lett. 143B (1984) 83 - 86

[13] A. Dobado and A. L. Maroto, Phys. Rev. D52 (1995) 1895 - 1901

[14] F. D. Mazzitelli, Phys. Rev. D45 (1992) 2814 - 2823

[15] H. J. de Vega and N. Sanchez, 'Lectures on String Theory in Curved Space-Times', in String Gravity and Physics at the Planck Energy Scale (Erice, 1995) 11 - 63

[16] A. L. Maroto and I. L. Shapiro, Phys. Lett. $\underline{414 B}$ (1997) 34 - 44

[17] A. Lukas, B. A. Ovrut and D. Waldram, Phys. Lett. B393 (1997) 65 - 71; 'The Cosmology of M-Theory and Type II Superstrings', hep-th/9802041; based on talks given at the Europhysics Conference on High Enrgy Physics, Jerusalem, Israel, August 1997 and at the XXXIIIrd Rescontres de Moriond, Fundamental Paramters in Cosmology, Les Arcs, Savoie, France, January, 1998

[18] A. P. Billyard, A. A. Coley, J. E. Lidsey, U. S. Nilsson, 'Dynamics of M-Theory Cosmology' (1999) hep-thy/99080102

[19] S. W. Hawking, 'Quantum Cosmology', Relativity, Groups and Topology II, Les Houches 1983, Session XL, edited by B. S. De Witt and R. Stora (North-Holland, Amsterdam, 1984) 333 - 379

S. W. Hawking, Nucl. Phys. B239 (1984) 257 - 276

[20] A. Vilenkin, Phys. Rev. D32 (1985) 2511 - 2548

[21] A. D. Linde, 'Inflation and Quantum Cosmology', Three Hundred Years of Gravitation, ed. by S. W. Hawking and W. Israel (Cambridge: Cambridge University Press, 1989) 604 - 630

A. D. Linde Inflation and Quantum Cosmology, ed. by R. H. Brandenburger (Boston: Academic Press, 1990) 
[22] N. A. Lemos, Phys. Rev. D53 (1996) 4275 - 4279

[23] J. E. Lidsey, Class. Quantum Grav. 11 (1994) 1211 - 1224; Phys. Rev. D49 (1994) $599-602$

[24] D. Clancy, J. E. Lidsey and R. Tavakol, Phys. Rev. D59 (1999) 063511

[25] R. Brustein and R. Madden, JHEP 9907 (1999) 006

[26] M. Gasperini, Int. J. Mod. Phys. $\underline{A 13}$ )(1998) 4779 - 4786

[27] R. Brustein, M. Gasperini and G. Veneziano, Phys. Rev. D55 (1997) 3882 - 3885

[28] M. D. Pollock, Nucl. Phys. B315 (1989) 528 - 540; Nucl. Phys. B324 (1989) 187 - 204

[29] M. D. Pollock, Int. J. Mod. Phys. A $\underline{7}(17)$ (1992) 4149 - 4165; Int. J. Mod. Phys. D $\underline{4}(3)(1995) 305-326$

[30] H. van Elst, J. E. Lidsey and R. Tavako, Class. Quantum Grav. 11 (1994) 2483 - 2497

[31] M. Ostrogradski, Mem. Acad. Imp. Sci. St. Petersburg, Serie VI (1850) 385

[32] E. T. Whittaker, A Treatise on the Analytic Dynamics of Particles Rigid Bodies (Cambridge: Cambridge University Press, 1927) pp. $265-267$

[33] M. D. Pollock, Mod. Phys. Lett. $\underline{A 12}$ (1997) 2057 - 2064

[34] P. H. M. Kersten (1985) Infinitesimal Symmetries: A Computational Approach, Ph. D. Thesis, Technische Hogeschool Dekanen 